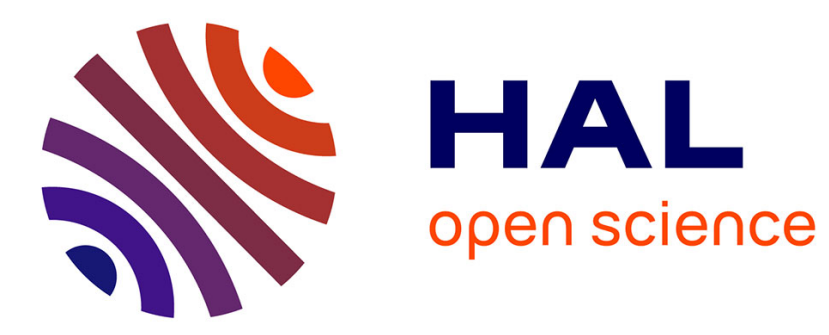

\title{
4D compressive sensing holographic imaging of small moving objects with multiple illuminations
}

Alexey Brodoline, Nitin Rawat, Daniel Alexandre, Nicolas Cubedo, Michel Gross

\section{- To cite this version:}

Alexey Brodoline, Nitin Rawat, Daniel Alexandre, Nicolas Cubedo, Michel Gross. 4D compressive sensing holographic imaging of small moving objects with multiple illuminations. Applied optics, 2019, 58 (34), pp.G127. 10.1364/AO.58.00G127 . hal-02321405

\section{HAL Id: hal-02321405 \\ https://hal.science/hal-02321405}

Submitted on 21 Oct 2019

HAL is a multi-disciplinary open access archive for the deposit and dissemination of scientific research documents, whether they are published or not. The documents may come from teaching and research institutions in France or abroad, or from public or private research centers.
L'archive ouverte pluridisciplinaire HAL, est destinée au dépôt et à la diffusion de documents scientifiques de niveau recherche, publiés ou non, émanant des établissements d'enseignement et de recherche français ou étrangers, des laboratoires publics ou privés. 


\title{
4D compressive sensing holographic imaging of small moving objects with multiple illuminations
}

\author{
Alexey Brodoline ${ }^{1,2,{ }^{*}}$, Nitin Rawat ${ }^{3}$, Daniel Alexandre ${ }^{1}$, Nicolas Cubedo $^{4}$, and Michel \\ Gross $^{1}$ \\ ${ }^{1}$ Laboratoire Charles Coulomb (L2C), Univ Montpellier, CNRS, Montpellier, France \\ ${ }^{2}$ Laboratoire Hubert Curien, UJM-Saint-Etienne, CNRS, Institut d'Optique Graduate \\ School, Saint-Etienne, France \\ ${ }^{3}$ Electrical and Computer Engineering Department, University of Connecticut, 371 \\ Fairfield Road, Storrs, Connecticut 06269, USA \\ ${ }^{4}$ Mécanismes Moléculaires dans les Démences Neurodégénératives (MMDN), Univ \\ Montpellier, INSERM, Montpellier, France \\ ${ }^{*}$ Corresponding author: alexey.brodoline@umontpellier.fr
}

\begin{abstract}
In previous work [Opt. Lett. 44, 2827 (2019)], we presented a method based on digital holography and orthogonal matching pursuit, which is able to determine the $3 \mathrm{D}$ positions of small objects moving within a larger motionless object. Indeed, if the scattering density is sparse in direct 3D space, compressive sensing algorithms can be used. The method was validated by imaging red blood cell trajectories in the trunk vascular system of a zebrafish (Danio rerio) larva. We give here further details on the reconstruction technique and present a more robust version of the algorithm based on multiple illuminations.
\end{abstract}

Citation: A. Brodoline, N. Rawat, D. Alexandre, N. Cubedo, and M. Gross, "4D compressive sensing holographic imaging of small moving objects with multiple illuminations," Appl. Opt. 58, G127-G134 (2019). 


\section{Introduction}

Digital holography (DH) enables to retrieve the amplitude and the phase of the optical field scattered by an object from the interference between this field and a reference beam [1]. The resulting interferogram, called the hologram, is recorded on an image sensor, making possible numerical reconstruction of the complex field [2].

For this purpose, the field on the sensor is backpropagated within a series of planes adjacent to the object. In this way, a spatial distribution of the field scattered in the vicinity of the object is obtained. Nevertheless, this 3D field does not fit the 3D distribution of the scattering density of the object.

As was shown in [3], a gold nanoparticle imaged by digital holographic microscopy (DHM) will give a cone of light after field backpropagation. The angle of the cone corresponds to the numerical aperture of the microscope objective, while its apex is located at the position of the gold bead. Holography, which is able to localize this apex, is thus well suited to track small particles, and was used for this purpose in a large number of works, especially flow imaging $[4,5,6,7]$.

Due to its phase retrieval capability, holography is a valuable complement to standard microscopic techniques and was widely applied to imaging of biological samples $[8,9,10]$. Quantitative phase imaging techniques are able to provide an estimate of the thickness or of the dry mass of a living cell $[11,12,13]$.

If a true $3 \mathrm{D}$ reconstruction of the object is required, techniques such as diffraction tomography should be considered. By recording a series of holograms for different directions of illumination and by performing a tomographic reconstruction, the $3 \mathrm{D}$ optical index distribution of the object can be computed $[14,15]$. However, because it requires to record a large number of holograms $(\geq 100)$, tomography is not well suited for the imaging of moving objects.

In the case where there is a basis in which the 3D scattering density is sparse enough, it is also possible to reconstruct a $3 \mathrm{D}$ image of the object from a single hologram by compressive sensing (CS) $[16,17]$. This point has been demonstrated by Brady et al. [18] who have imaged by holography an immobile object whose representation is sparse in the wavelet basis. Since this work, DH coupled to the CS has given rise to many developments. We can mention compressive in-line holography [19], compressive Fresnel holography [20], off-axis frequency shifting holography [21], off-axis holography of diffuse objects [22], video-rate microscopic tomography [23], or incoherent holographic microscopy [24]. Recently, a combination of compressive holography and coded exposure enabled the reconstruction of $3 \mathrm{D}$ videos of small sparse objects [25].

In this work, we focused on 3D imaging of blood circulation in zebrafish (Danio rerio) larvae, which implies to localize in space and in time the positions of the red blood cells (RBCs). Due to the fact that their refractive index is close to the one of the surrounding medium, the cone of the scattered light is narrow. For this reason, the RBCs can not be precisely localized with classical holographic backpropagation. In [26], we proposed a technique based on a specific holographic setup and a "cleaning" algorithm, which enabled to compute the positions of the RBCs in 3D, as well as the shape of the blood vessels. Nevertheless, the drawback of this algorithm was the relatively long computing time. In [27], we described an algorithm which enabled to speed up calculations by a factor of $\sim 500$, based on compressive sensing.

Indeed, RBCs can be viewed as small scattering objects that move within a larger motionless object (the anesthetized zebrafish larva). In the basis of the direct 3D space (i.e. $x, y, z$ ), their 3D scattering density is sparse, which allows to apply compressive sensing reconstruction methods. In this specific case, the use of sparsifying operators [28] is not required. We used an algorithm based on orthogonal matching pursuit (OMP). The case of objects composed of point sources was also studied by Liu et al. [29], where a more complex reconstruction algorithm was proposed based on similar compact sensing matrix computation.

By performing calculations from a small number of holograms, we were able to obtain the 3D positions of the RBCs at one camera frame. By performing a series of reconstructions from a long sequence of holograms, we got movies of the 3D positions of RBCs, and by averaging in time, we determined the shape of 
the blood vessels from the trajectories of the RBCs.

In this paper, we will give further details on the experimental configuration and the reconstruction procedure. We will, in particular, describe a method for the localization of the RBCs that uses the fact that the object is illuminated by several beams, which results in a more robust reconstruction algorithm.

\section{Experimental setup}

The experiment is carried out in an off-axis configuration similar to previous work [26]. To increase the angular diversity of illumination, the sample was imaged with a higher NA microscope objective $(20 \mathrm{x} / \mathrm{NA}=0.5$ water immersion, Zeiss $)$ and with three illumination beams, instead of $\mathrm{NA}=0.3$ and two illuminations in [26]. The holographic signal was acquired by a Mikrotron EoSens CL camera $(1280 \times 1024$ pixels, $14 \mu \mathrm{m}, 12$ Bits, 200 frames/s $)$. The laser diode power at the sample was less than 1 $\mathrm{mW}$ at $\lambda=662 \mathrm{~nm}$.

The experimental setup is depicted in Fig. 1. The illumination beam $E_{I L}$ generated by a laser diode is divided into three using beam splitters (BS) and mirrors $(\mathrm{M})$. The object is thus illuminated from three different directions. The intensities of the beams can be equalized with neutral density filters. The scattered light $E$ is collected by the microscope objective (MO) and imaged on the camera, in plane $C^{\prime}$, where it interferes with the reference field $E_{R}$. The combining $\mathrm{BS}$ is tilted with an angle $\theta$ to perform off-axis holography. The camera records the resulting interferogram $I=\left|E_{C^{\prime}}+E_{R}\right|^{2}$, where $E_{C^{\prime}}$ is the field scattered by the object in the camera plane $C^{\prime}$.

We have validated the technique by imaging the microcirculation in zebrafish (Danio rerio) larvae. Unpigmented larvae were obtained from spontaneous spawning of casper young adult fish pairs and grown at $28.5^{\circ} \mathrm{C}$. They were anesthetized with tricaine at five days and mounted in lateral view in $1 \%$ low melting point agarose in a $35 \mathrm{~mm}$ glass ibidi bottom petri dish. The trunk blood flow was imaged immediately. Animals were handled according to standard procedures [30].

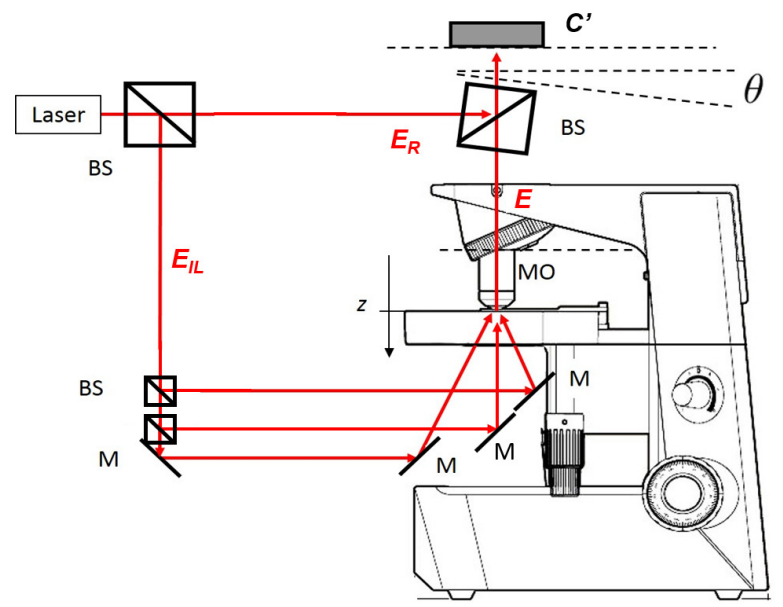

Figure 1: Triple illumination off-axis holographic microscopy setup. BS, beam splitters; M, mirrors; MO, microscope objective; $\theta$, off-axis angle; $C^{\prime}$, camera plane; $E_{I L}$, illumination beam; $E$, scattered object field; and $E_{R}$, reference field.

\section{Selection of the moving ob- jects}

The zebrafish larva is an optically thick object composed of tissues, organs and bones scattering light, which results in a signal irrelevant for our applications. In order to select the moving RBCs and eliminate this background, we considered sequences of interferograms $I_{m}$ with $m=0,1,2 \ldots$ corresponding to successive camera frames. The resulting hologram $H_{C^{\prime}}$ in the camera plane $C^{\prime}$ is then obtained by combining the $I_{m}$ with coefficients whose sum is zero:

$$
H_{C^{\prime}}(x, y)=\sum_{m} \eta_{m} I_{m}(x, y) \text { with } \sum_{m} \eta_{m}=0
$$

For example, $H_{C^{\prime}}=\left(I_{m}+I_{m-1}\right)-\left(I_{m-2}+I_{m-3}\right)$, and $\eta_{m}=(1,1,-1,-1)$.

In this way, the immobile background is subtracted, and the RBCs whose positions changed across the frames remain in the hologram. The number of $I_{m}$ and the interval between them can be changed in order to increase the signal amplitude. 


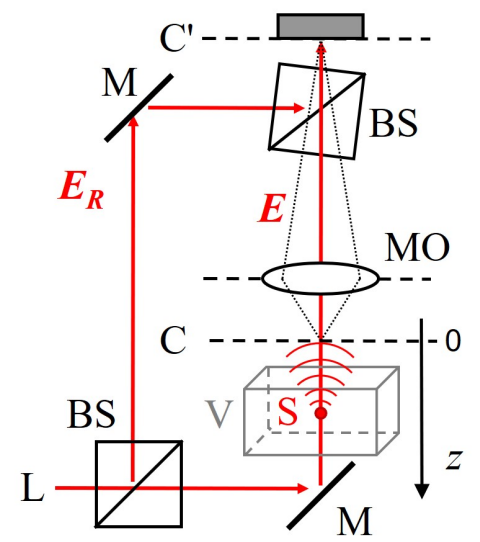

Figure 2: Typical off-axis holographic microscopy setup. L, laser; C', camera; BS, beam splitter; $\mathrm{M}$, mirror; $\mathrm{MO}$, microscope objective; $\mathrm{C}$, conjugate plane of plane C' by objective MO; V, imaged volume; S, a scattering object; $E$, object field; and $E_{R}$, reference field.

This approach is very similar to the one of Schwerte and Pelster [31] who highlighted blood circulation in zebrafish larvae by calculating differences of successive frames in white light video movies. This approach was also used with holography in [32].

In practice, we considered combinations of six holograms, according to the following equation (with $N=6)$ :

$$
H_{C^{\prime}}(x, y)=\sum_{k=0}^{N-1} \sin \left(\frac{2 \pi k}{N}\right) I_{m}(x, y) .
$$

If we consider a higher $N$, we will average on more frames, which will result in more signal but can as well increase the noise.

\section{Formulation of the compres- sive sensing equations}

A simplified outline of the setup with a single illumination is depicted in Fig. 2. The imaged objects are located in a volume $\mathrm{V}$ and illuminated by a laser diode $\mathrm{L}$. The scattered light $E$ is collected by the $\mathrm{MO}$ and imaged on the camera, in plane $C^{\prime}$. It interferes with the reference field $E_{R}$ to form the hologram $I=\left|E_{C^{\prime}}+E_{R}\right|^{2}$, where $E_{C^{\prime}}$ is the field scattered by the objects in the camera plane. Since the experiment is carried out in off-axis geometry, by filtering in the Fourier space [33], we can select the +1 order component of the hologram, corresponding to the $E_{C^{\prime}} E_{R}^{*}$ term.

By considering that the reference field is a flat field $\left(\left|E_{R}\right| \simeq 1\right)$, we thus calculate the field $E_{C^{\prime}}$ and eliminate the phase effects related to eventual aberrations of the MO [34], to the off-axis configuration and to the curvature of the reference wave [35]. We get therefore the field $E_{C}(x, y)$ in the plane $C$, conjugate of the plane $C^{\prime}$ by the MO. We get as well the field $\tilde{E}_{C}$ in the Fourier space, $\tilde{E}_{C}\left(k_{x}, k_{y}\right)=\mathrm{FT}\left[E_{C}(x, y)\right]$, where FT is the discrete Fourier transform operator.

From now, we can combine several holograms in order to eliminate the background and select only the moving RBCs. In the following, the fields $E_{C^{\prime}}$, $E_{C}$ and their Fourier transforms designate the fields scattered by the moving objects only.

The RBCs that scatter light, act like small sources of field $S(x, y, z)$ located in volume $\mathrm{V}$ [Fig. 2]. According to angular spectrum propagation equations [36], the field $E_{C}(x, y)$ radiated by the sources $S$ in the plane $C$ at $z=0$ is $E_{C}(x, y)=\sum_{z} \mathrm{FT}^{-1} \exp \left(+i k_{z} \cdot z\right) \mathrm{FT}[S(x, y, z)]$, (where $\mathrm{FT}^{-1}$ is the inverse Fourier transform operator). With the linearity of the Fourier transform, we get the expression of the scattered field spectrum in the camera conjugate plane:

$$
\tilde{E}_{C}\left(k_{x}, k_{y}\right)=\sum_{z} \exp \left(+i k_{z} . z\right) \operatorname{FT}[S(x, y, z)],
$$

where $k_{z}$ is the $z$ component of the wavevector $\mathbf{k}$, i.e., $k_{z}=\sqrt{k^{2}-k_{x}^{2}-k_{y}^{2}}$ with $k=|\mathbf{k}|=2 \pi n_{m} / \lambda$, where $\lambda$ is the wavelength in vacuum and $n_{m} \simeq 1.33$ the refractive index of the medium.

Equation (3) can be written as a linear system of equations that connects the $3 \mathrm{D}$ field scattered by the objects to the field spectrum in the camera conjugate plane:

$$
\mathbf{u}=A \mathbf{v},
$$


where $\mathbf{v} \equiv S(x, y, z)$ and $\mathbf{u} \equiv \tilde{E}_{C}\left(k_{x}, k_{y}\right)$ are vectors of dimensions $768 \times 768 \times 160$ and $768 \times 768$, respectively, and $A$ is a matrix of dimension $768^{4} \times 160$ [see Fig. 3(a)].

This system is strongly under-determined since the dimensions of vectors $\mathbf{u}$ and $\mathbf{v}$ correspond to the number of pixels of the calculation grid in plane $C$ and the number of voxels of the volume $\mathrm{V}$, respectively. The compression ratio $(\mathrm{CR})$ between the vectors is large, since it corresponds to the number of planes $z$ considered for the $3 \mathrm{D}$ reconstruction (i.e., $\mathrm{CR}=160$ ).

We should note that the matrix $A$ is too large to be calculated. To compute $\mathbf{u}=A \mathbf{v}$, (3) must be used. Similarly, to compute $\mathbf{v}=A^{\dagger} \mathbf{u}$, we must use (5) for each $z$ :

$$
S(x, y, z)=\mathrm{FT}^{-1}\left[\exp \left(-i k_{z} . z\right) \tilde{E}_{C}\left(k_{x}, k_{y}\right)\right] .
$$

The moving RBCs represent a very small part of the imaged volume, and the vector $\mathbf{v}$ is highly sparse in the basis of the positions $x, y, z$. It is then possible to solve (4) iteratively using compressive sensing.

\section{Orthogonal matching pursuit algorithm}

The CS method we used here is based on the orthogonal matching pursuit (OMP) [37]. It is described in detail in our previous article [27]. Let us recall the main steps of the algorithm. It can be schematized by the equation:

$$
\mathbf{u}^{(n+1)}=\mathbf{u}^{(n)}-a_{n} A \mathrm{~T}^{(n)} A^{\dagger} \mathbf{u}^{(n)},
$$

where $n$ is the iteration index. The initialization $\mathbf{u}^{(1)}=\mathbf{u} \equiv \tilde{E}_{C}$ is done with the object field in the Fourier space obtained from the +1 order of the recorded hologram after phase corrections and background subtraction.

The algorithm calculates iteratively the $3 \mathrm{D}$ sources $\mathbf{v}^{(n)}=A^{\dagger} \mathbf{u}^{(n)}$, and the 2D field $A \mathbf{v}^{(n)}$ radiated by the sources, by selecting within the sources $\mathbf{v}^{(n)}$ the brightest ones with the operator $\mathrm{T}^{(n)}$. The $2 \mathrm{D}$ field radiated by the selected sources (i.e. $A \mathrm{~T}^{(n)} A^{\dagger} \mathbf{u}^{(n)}$ ) is subtracted from the $2 \mathrm{D}$ field $\mathbf{u}^{(n)}$ in order to get (a)

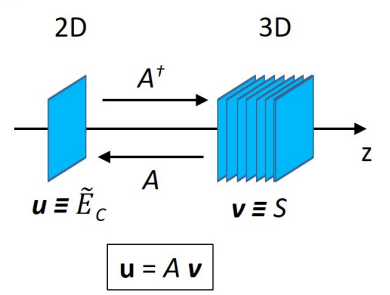

(b)

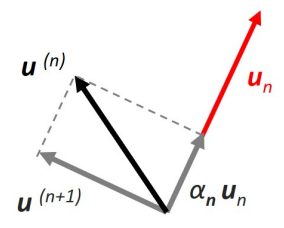

Figure 3: (a) Schematic representation of the transformations. Matrix $A$ describes the emission by the 3 D sources $\mathbf{v} \equiv S$ resulting in a $2 \mathrm{D}$ field in Fourier space $\mathbf{u} \equiv \tilde{E}_{C}$. Matrix $A^{\dagger}$ represents the $3 \mathrm{D}$ field reconstruction from the $2 \mathrm{D}$ field in the camera conjugate plane. (b) Normalization procedure diagram: $\mathbf{u}^{(n)}$ is the hologram at the iteration $n, \mathbf{u}_{n}$ the field radiated by the sources selected at iteration $n, a_{n}$ the normalization coefficient, and $\mathbf{u}^{(n+1)}$ the hologram at the iteration $n+1$, orthogonal to $\mathbf{u}_{n}$.

the 2D field for the next iteration $\mathbf{u}^{(n+1)}$. In this way, the 2D hologram in Fourier space is cleaned at each iteration, while the positions of the selected sources are saved.

We should remark that in order to optimize and stabilize the cleaning procedure, the selected sources $\mathrm{T}^{(n)} \mathbf{v}^{(n)}$ are multiplied by a normalization factor $a_{n}$. It is adjusted so that the resulting vector $\mathbf{u}^{(n+1)}$ remains orthogonal to the vector $\mathbf{u}_{n}=A \mathrm{~T}^{(n)} A^{\dagger} \mathbf{u}^{(n)}$ radiated by the selected sources [Fig. 3(b)]. The energy of the 2D field $\left|\mathbf{u}^{(n+1)}\right|^{2}$ is thus minimized after cleaning.

To achieve the condition of orthogonality, we have to satisfy $\left(\mathbf{u}^{(n)}-a_{n} \mathbf{u}_{n}\right) \cdot \mathbf{u}_{n}=0$, where $\boldsymbol{~}$ is the scalar product. We get

$$
a_{n}=\frac{\mathbf{u}_{n} \cdot \mathbf{u}^{(n)}}{\left|\mathbf{u}_{n}\right|^{2}} .
$$

At each iteration step $n$, the total energy (summed over all pixels) remaining in the 2D field $\left|\mathbf{u}^{(n)}\right|^{2}$ decreases. When this energy becomes sufficiently low, e.g., when it represents only $20 \%$ of the initial energy, the iterative process stops. The final 3D distribution 
$\mathbf{v}$ of the sources is given by the summation of the sources selected at each iteration:

$$
\mathbf{v}=\sum_{n} a_{n} \mathrm{~T}^{(n)} A^{\dagger} \mathbf{u}^{(n)} .
$$

It represents the $3 \mathrm{D}$ distribution of the $\mathrm{RBCs}$ at one camera frame.

\section{Operator $T^{(n)}$ for the selec- tion of the sources}

The reconstruction speed and quality depend of the operator $T^{(n)}$ that selects at each iteration $n$ the brightest sources. There are several ways to define this operator. In [27], the criterion to select the RBCs was the maximum of the energy of the complex field. A second manner to proceed is to use the energy correlation between the three laser beams illuminating the object. Let us give here more details on these methods of selection of the RBCs.

\subsection{Selection of the sources based on energy}

In order to find the positions of the RBCs, we can calculate the energy of the complex 3D field and look for its maxima. Indeed, the RBCs are located at the intersections of apexes of the cones of scattered light there energy is maximum. We should remark that the localization is possible only due to the multiple illumination. Since the refractive index of RBCs is very close to the one of the surrounding medium, the angle of the scattering cones is small, and thus the localization with a single beam would not be precise enough.

Let us consider the Fourier transform of the off-axis hologram, represented on a $1280 \times 1280$ calculation grid [Fig. 4(a)]. The zero grating order is on the center, while the +1 and -1 orders that represent the rear pupil of the microscope objective are on the top and on the bottom, respectively. The three bright spots inside the pupil correspond to the ballistic light of the illumination beams. Note that the objective has a phase ring which can be seen on the figure.
Since it does not overlap with the beams, it has no effect on our reconstructions.

The +1 grating order signal (which corresponds to $\left.\tilde{E}_{C}\left(k_{x}, k_{y}\right)\right)$ was selected by cutting a disk of radius 340 pixels within the Fourier space. This disk was copied on a $768 \times 768$ calculation grid [Fig. 4 (b)] The zero order was filtered by a circular mask of radius 170 to avoid signal mixing. The center of the pupil with the phase ring was as well removed with a disk of radius 195 pixels.

The algorithm starts with the filtered +1 order $\mathbf{u}^{(1)} \equiv \tilde{E}_{C}\left(k_{x}, k_{y}\right)$. At each iteration step $n$, after the calculation of the $3 \mathrm{D}$ field $\mathbf{v}^{(n)}=A^{\dagger} \mathbf{u}^{(n)}$ with (5), the energy of the sources $\left|\mathbf{v}^{(n)}\right|^{2} \equiv\left|S^{(n)}(x, y, z)\right|^{2}$ is calculated for each voxel $(x, y, z)$. Then a threshold of energy $\mathcal{E}_{t h}^{(n)}$ is chosen, e.g. $\mathcal{E}_{t h}^{(n)}=0.8 \mathcal{E}_{\text {max }}^{(n)}$, where $\mathcal{E}_{\text {max }}^{(n)}$ is the maximum of the vector $\left|\mathbf{v}^{(n)}\right|^{2}$.

Afterwards, the operator $T_{\mathcal{E}}^{(n)}$ selects the voxels $\left(x^{\prime}, y^{\prime}, z^{\prime}\right)$ whose energy is above the threshold $\mathcal{E}_{t h}^{(n)}$ and adds to these voxels the voxels located within a predefined radius (e.g., $r_{R B C}=4$ pixels) in the same $z=z^{\prime}$ plane. The selected sources $\mathrm{T}_{\mathcal{E}}^{(n)} A^{\dagger} \mathbf{u}^{(n)} \equiv$ $S_{n}(x, y, z)$ are thus

- $S_{n}(x, y, z)=S^{(n)}(x, y, z)$ if $\left|S^{(n)}\left(x^{\prime}, y^{\prime}, z^{\prime}\right)\right|^{2}>$ $\mathcal{E}_{t h}^{(n)}, \sqrt{\left(x^{\prime}-x\right)^{2}+\left(y^{\prime}-y\right)^{2}} \leq r_{R B C}$, and $z=z^{\prime}$,

- $S_{n}(x, y, z)=0$ otherwise.

Two criteria can be used to terminate the algorithm. In [27], the stopping criterion was the value of the total energy of the hologram. When it becomes lower than $20 \%$ of the initial energy, i.e., if $\left|\mathbf{u}^{(n)}\right|^{2}<0.2\left|\mathbf{u}^{(1)}\right|^{2}$, the algorithm ends. Here, we considered the ratio between the maximum energy at the current iteration and the maximum at the first iteration. When it becomes sufficiently low, e.g., if $\mathcal{E}_{\max }^{(n)} / \mathcal{E}_{\max }^{(1)}<0.06$, the calculation will stop.

In practice, the second criterion is reached before the first. The final $3 \mathrm{D}$ sources $\mathbf{v} \equiv S(x, y, z)$ are then obtained by a summation of all the detected sources and by taking into account the normalization coefficients:

$$
S(x, y, z)=\sum_{n} a_{n} S_{n}(x, y, z) .
$$



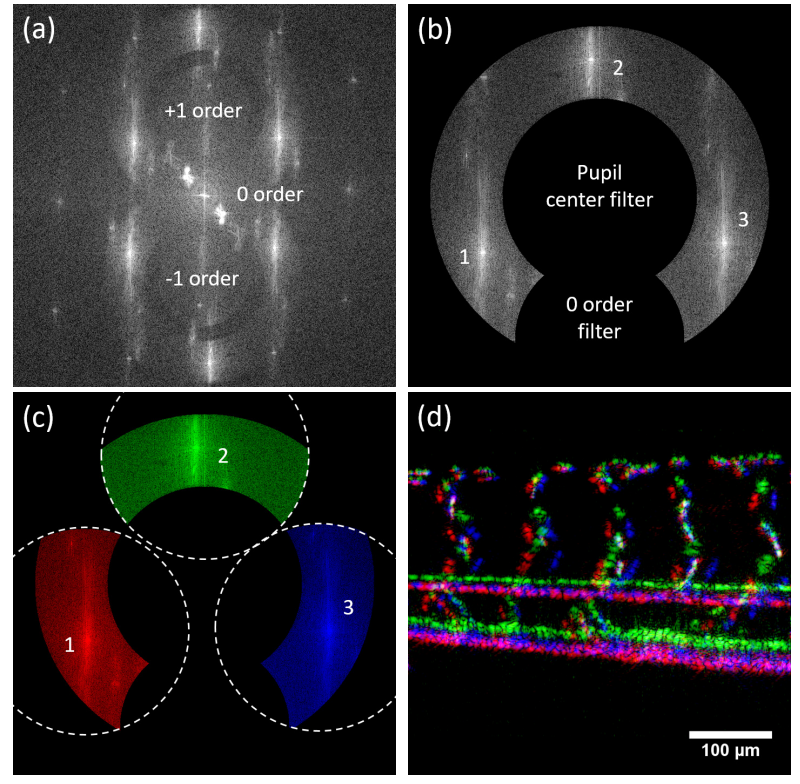

Figure 4: (a) Fourier transform of the off-axis hologram. The bright spots inside the +1 and -1 orders correspond to the three illumination beams. (b) Crop of the +1 order of the hologram, filtering of the center of the pupil and of the zero order. (c) Separation of the three illumination beams inside the +1 order with circular masks (red, green and blue). (d) Intensity of the three fields (red, green and blue) reconstructed in the camera conjugate plane.

\subsection{Selection of the sources with mul- tiple illuminations}

A second approach is to consider separately the three laser beams illuminating the object from different directions. The separation can be performed in the Fourier space, while the +1 order of the hologram is selected.

Figure 4(c) shows the crop of the +1 order, where three circular masks (in red, green and blue) are applied to select the light scattered by the illumination beams. The bright peaks correspond to ballistic light, while the surrounding halo constitutes scattered light. The complex 3D field can then be computed for the three beams separately, giving the vectors $\mathbf{v}^{(n, i)} \equiv S^{(n, i)}(x, y, z)$ with $i=1,2,3$. The intensities of the three fields in the direct space in plane $C$ are represented in Fig. 4(d).

The principle of the OMP algorithm is the same as previously. Nevertheless, the transforms associated with $A$ and $A^{\dagger}$ are applied to the three fields separately, and the reconstruction of the vectors $\mathbf{u}^{(n, i)}$ (with $i=1,2,3$ ) in the Fourier space with (3) has to take into account the selection masks.

Another major change is the criterion of selection of the sources $S_{n}(x, y, z)$ with the operator $T_{\mathcal{C}}^{(n)}$. After the calculation of the vectors $S^{(n, i)}$ in the object space, we calculate for each voxel the product of the energies of the three fields, which we call the correlation $\mathrm{Corr}^{3}$, similar to what was done in [26]:

$\begin{aligned} \operatorname{Corr}_{n}^{3}(x, y, z) & =\left|S^{(n, 1)}(x, y, z)\right|^{2} \\ & \times\left|S^{(n, 2)}(x, y, z)\right|^{2} \times\left|S^{(n, 3)}(x, y, z)\right|^{2}\end{aligned}$

Since we have a product of three energies, to get an equivalency with the previous method, we should consider the cube root $\operatorname{Corr}_{n}=\sqrt[3]{\operatorname{Corr}_{n}^{3}}$ at each pixel. We define then a threshold $\mathcal{C}_{t h}^{(n)}$, based on the maximum value of $\operatorname{Corr}_{n}$, e.g., $\mathcal{C}_{t h}^{(n)}=0.8 \operatorname{Corr}_{n, \text { max }}$. The operator $T_{\mathcal{C}}^{(n)}$ selects the voxels $\left(x^{\prime}, y^{\prime}, z^{\prime}\right)$ whose correlation is above the threshold $\mathcal{C}_{t h}^{(n)}$ and adds to these voxels the voxels located within a predefined radius (e.g., $r_{R B C}=4$ pixels) in the same $z=z^{\prime}$ plane. The selected sources $\mathrm{T}_{\mathcal{C}}^{(n)} A^{\dagger} \mathbf{u}^{(n)} \equiv S_{n}(x, y, z)$ are thus

- $S_{n}(x, y, z)=\left\langle S^{(n, i)}(x, y, z)\right\rangle_{i}$ if $\operatorname{Corr}_{n}\left(x^{\prime}, y^{\prime}, z^{\prime}\right)>$ $\mathcal{C}_{t h}^{(n)}, \sqrt{\left(x^{\prime}-x\right)^{2}+\left(y^{\prime}-y\right)^{2}} \leq r_{R B C}$, and $z=z^{\prime}$,

- $S_{n}(x, y, z)=0$ otherwise,

where $\langle.\rangle_{i}$ represents the average among the three fields.

The remaining calculations such as the cleaning of $\mathbf{u}^{(n)}$ with the use of the coefficient $a_{n}$ and the summation of all the sources after the last iteration are the same as for the energy. 


\section{Reconstructions analysis}

\subsection{D images of zebrafish microcir- culation}

The CS calculations were conducted on a $768 \times 768 \times$ 160 tridimensional grid and on 256 camera frames in approximately $2 \mathrm{~h}$ using GPU (NVidia GTX Titan Xp) and CUDA libraries (cuFFT, cuBlas...). Voxel size was $0.6 \mu \mathrm{m}$ in $x$ and $y$, and $1.8 \mu \mathrm{m}$ in $z$. The $3 \mathrm{D}$ visualizations were performed with a homemade CUDA program based on an NVidia code example (VolumeRender).

Figure 5(a) shows the 3D positions of the RBCs at one camera frame obtained from a combination of six holograms modulated by a sinus and using the operator $T_{\mathcal{C}}^{(n)}$ that selects the brightest sources taking account of the correlation among the three illumination beams. It can be noticed that each RBC produces two bright spots due to the image subtraction. A complete $360^{\circ}$ rotation can be seen in (Visualization 1).

Figures 5(b) and 5(c) show the 3D images of the shape of the blood vessels, obtained by averaging the positions of the RBCs over 256 camera frames. The reconstructed vascular system compares favorably to images obtained by confocal microscopy [38]. We should remark that we do not reconstruct the blood vessels walls like it can be done in fluorescence microscopy. Nonetheless, the shape of the main trunk vessels is well reconstructed. A complete $360^{\circ}$ rotation of the vascular system can be found in (Visualization 2).

In order to visualize the $3 \mathrm{D}$ motion of the RBCs inside the blood vessels, we superimposed the nonaveraged and averaged reconstructions [Fig. 5(d)]. A $360^{\circ}$ rotation of this sequence of $3 \mathrm{D}$ images is shown in Visualization 3. In the movie, the direction of blood flow in the dorsal aorta, caudal vein, intersegmental vessels and dorsal longitudinal anastomotic vessels can be determined. Since the camera has a relatively high frame rate (200 images/s), the RBCs motion appears fluid.

Figure 6(b) shows the reconstruction obtained considering only the energy of the hologram (i.e., with the operator $\left.T_{\mathcal{E}}^{(n)}\right)$. It can be compared with the
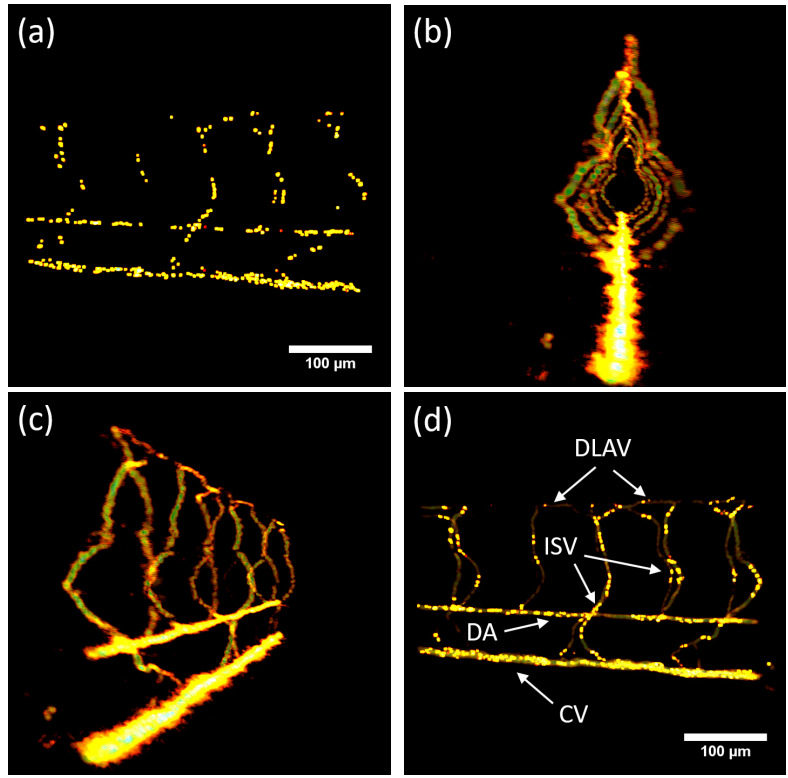

Figure 5: (a) Representation of RBCs considering a single 3D reconstruction at one camera frame. View at $0^{\circ}$. (b,c) Representations of the vascular system considering a summation of a sequence of 256 holograms. Views at $96^{\circ}$ and $120^{\circ}$. (d) The RBCs positions are superimposed with the shape of the blood vessels. Dorsal aorta (DA), caudal vein $(\mathrm{CV})$, intersegmental vessels (ISV) and dorsal longitudinal anastomotic vessels (DLAV) can be clearly seen. View at $0^{\circ}$. Reconstructions performed with operator $T_{\mathcal{C}}^{(n)}$ and a selection threshold $\mathcal{C}_{t h}^{(n)}=0.8 \operatorname{Corr}_{n, \max }$. For a $360^{\circ}$ rotation see Visualizations in supplement.

reconstruction that considers the three illumination beams [Fig. 6(a)]. We can remark that the difference is very small. Nevertheless, the image obtained with $T_{\mathcal{E}}^{(n)}$ seems to be noisier, with artifacts along the field propagation direction $z$ (white arrows).

\subsection{Convergence of the algorithm}

Figure 7(a) shows the mean decrease in total energy of the hologram with time for operators $T_{\mathcal{E}}^{(n)}$ and $T_{\mathcal{C}}^{(n)}$. The algorithm converges in fewer iterations when the correlation among the three illuminations is 

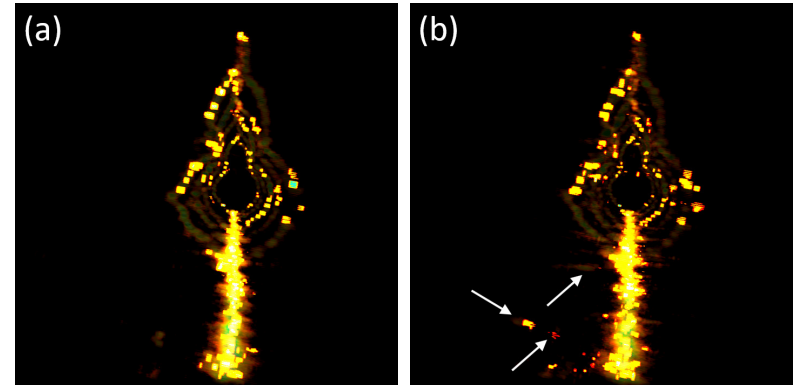

Figure 6: Comparison between the reconstructions with the two operators $T^{(n)}$ : (a) Reconstruction using triple illumination with $T_{\mathcal{C}}^{(n)}$ and selection threshold $\mathcal{C}_{t h}^{(n)}=0.8 \operatorname{Corr}_{n, \max }$. (b) Reconstruction based on energy with $T_{\mathcal{E}}^{(n)}$ and selection threshold $\mathcal{E}_{t h}^{(n)}=$ $0.8 \mathcal{E}_{\max }^{(n)}$. Views at $96^{\circ}$. White arrows: reconstruction artifacts.

considered. Nevertheless, the computing time of one iteration is about twice longer, and therefore the total calculation times remain equivalent for both cases. Figure 7(b) shows the mean decrease of the ratio between the maximum energy (or correlation) at the current iteration by the maximum energy (or correlation) at the first iteration. The algorithm stops when this ratio is smaller than 0.06 .

Figures $7(\mathrm{a})$ and $7(\mathrm{~b})$ show as well the cases where the chosen threshold value is too low $\left(\mathcal{E}_{t h}^{(n)}=0.5 \mathcal{E}_{\max }^{(n)}\right.$ and $\left.\mathcal{C}_{t h}^{(n)}=0.5 \operatorname{Corr}_{n, \max }\right)$. In this case, the algorithm converges very fast, since an important amount of energy is removed at each iteration. Nevertheless, this reduces the quality of the $3 \mathrm{D}$ reconstructions. In Fig. 7(b), in the case where the reconstruction is based only on energy, the ratio variation is not stable and increases at some iterations. The second algorithm is more robust and does not present such non-uniformity.

Figure 8 shows the reconstructions obtained with low thresholds (i.e., 0.5). In the case where only the energy was considered, the 3D image is incomplete. The dorsal vessels where the blood flow was not significant are not reconstructed. High-energy artifacts elongated in the $z$ direction are as well present (white arrows).

\section{(a)}

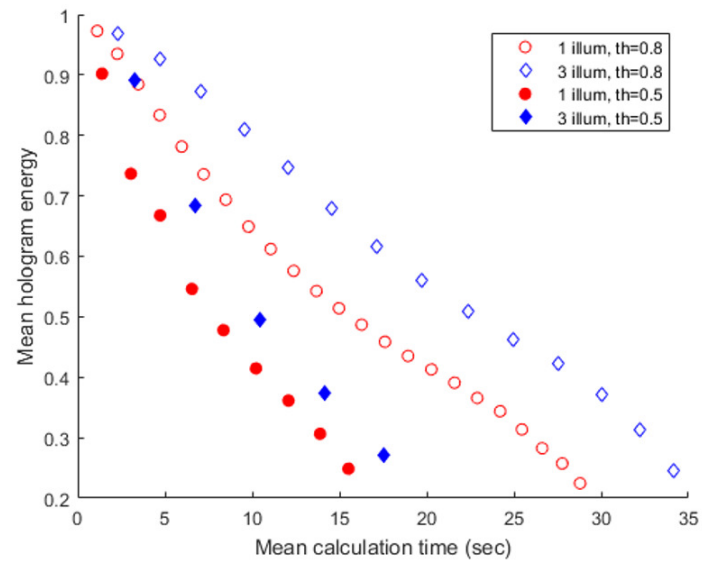

(b)

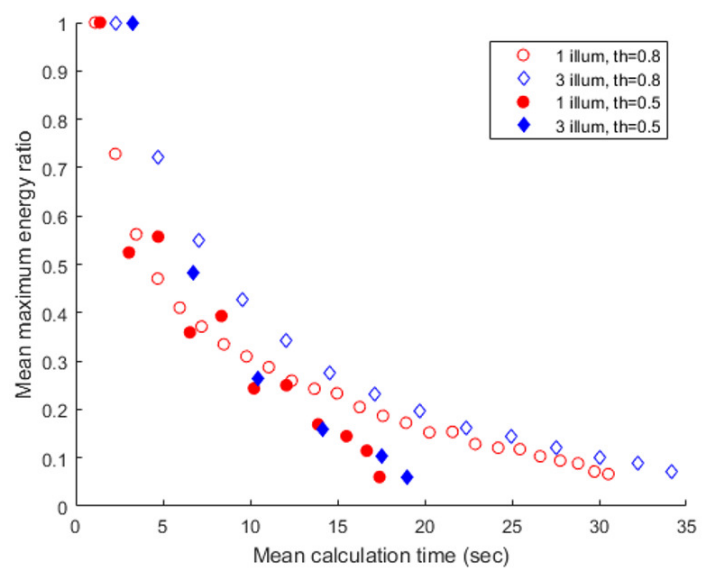

Figure 7: (a) Mean decrease in energy of the hologram with time. (b) Temporal variation of the ratio between the mean maximum energy or correlation and its value at the first iteration. The averages of energy, correlation, and time are performed on 256 images. Plots are given for the cases where $T^{(n)}$ considers total energy (red circles) or correlation between the three beams (blue diamonds) as well as for two selection thresholds: 0.8 (empty) and 0.5 (full). Each point corresponds to one iteration of the algorithm. 

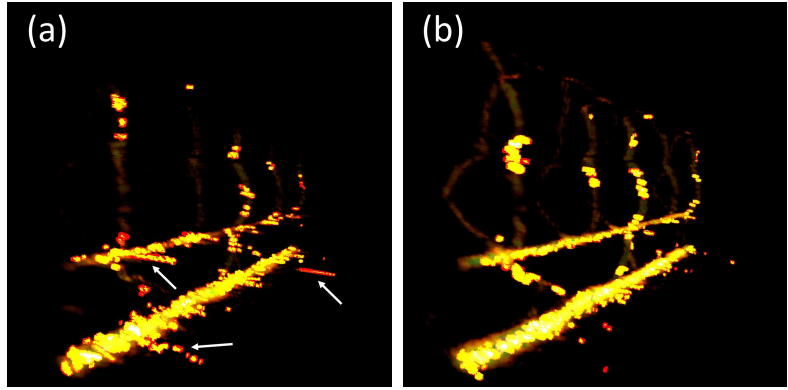

Figure 8: (a) Reconstruction by selection of the sources based on energy with $T_{\mathcal{E}}^{(n)}$ and a threshold $\mathcal{E}_{t h}^{(n)}=0.5 \mathcal{E}_{\max }^{(n)}$. (b) Reconstruction by selection of the sources based on the correlation between the three beams with $T_{\mathcal{C}}^{(n)}$ and a threshold $\mathcal{C}_{t h}^{(n)}=0.5 \mathcal{C}_{\max }^{(n)}$. White arrows: reconstruction artifacts.

The reconstruction with the three illumination beams [Fig. 8(b)] is more robust, and the shape of the vascular system is nonetheless retrieved. In some frames however, the least bright $\mathrm{RBCs}$ in the dorsal vessels are not reconstructed, which results in a blinking image along the frames.

\section{Conclusion}

In this paper, we presented a reconstruction procedure based on CS and a specific DHM setup using a triple illumination of the object. This technique is able to efficiently retrieve the $3 \mathrm{D}$ positions of the RBCs in the zebrafish larva vascular system, since they represent small scattering objects moving in a large motionless volume. The reconstruction of the $3 \mathrm{D}$ positions of the RBCs as well as the shape of the perfused vascular system enables to perform in-vivo imaging of microcirculation.

We compared two methods of selection of the RBCs; one is based on the maximum of the energy of the hologram, while the second considers the correlations in energy between the three illumination beams. The last one takes explicitly into account the fact that the object is illuminated by three beams. It appears to be more robust to ill choices of threshold parame- ters and gives less noisy results.

We should remark that several parameters influence the amplitude of the reconstruction noise. For example, in order to eliminate the background, we considered combinations of six holograms with sinus coefficients $\eta_{m}$. By combining more images, we will average on more frames and get therefore more signal with, at the same time, the risk of increasing noise. We could as well consider complex exponential coefficients $\eta_{m}$. In this case, the RBCs would appear more elongated. The influence of the stopping criterion should also be taken into account. If the chosen threshold is too small, irrelevant low energy signal will be selected, resulting in reconstruction artifacts in the image.

The basis used for CS calculations is the direct 3D space $(x, y, z)$ and since the object is sparse in this basis, the OMP method works quite well. The dictionary is simply composed of point sources at every $(x, y, z)$ coordinate. Since the object is illuminated by several beams, the reconstruction does not require an orthogonal basis where every pair of voxels would have uncorrelated holograms. An optimized basis could be learned, but it would necessitate a large number of test holograms to constitute the dictionary as in $[39,40]$.

We could also try more complex reconstruction algorithms on our data, in particular the one proposed by Liu et al. [29], or regularized algorithms such as LASSO [41], thresholding algorithms such as FISTA [42], and bayesian algorithms [43]. We have nevertheless to take into account memory issues, since the dimensions of our sensing matrix are very large.

\section{Funding}

LabEx Numev (ANR-10-LABX-20: Micro Holo 4D); Centre National de la Recherche Scientifique (CNRS) (Défi: instrumentation aux limites 2018).

\section{Acknowledgments}

The authors acknowledge Corinne Fournier and Laurent Daudet for fruitful discussions. Portions of this 
work were presented at the OSA Digital Holography and 3-D Imaging conference in 2019, paper Th2B.7 [44].

\section{References}

[1] D. Gabor. A new microscopic principle. Nature, 161:777-778, 1948.

[2] U. Schnars and W. Jüptner. Direct recording of holograms by a ccd target and numerical reconstruction. Applied optics, 33(2):179-181, 1994.

[3] M. Atlan, M. Gross, P. Desbiolles, E. Absil, G. Tessier, and M. Coppey-Moisan. Heterodyne holographic microscopy of gold particles. Optics letters, 33(5):500-502, 2008.

[4] C. Buraga-Lefebvre, S. Coëtmellec, D. Lebrun, and C. Ozkul. Application of wavelet transform to hologram analysis: three-dimensional location of particles. Optics and Lasers in Engineering, 33(6):409-421, 2000.

[5] F. Soulez, L. Denis, C. Fournier, E. Thiébaut, and C. Goepfert. Inverse-problem approach for particle digital holography: accurate location based on local optimization. JOSA A, 24(4):1164-1171, 2007.

[6] L. Tian, N. Loomis, J.A. Domínguez-Caballero, and G. Barbastathis. Quantitative measurement of size and three-dimensional position of fastmoving bubbles in air-water mixture flows using digital holography. Applied optics, 49(9):15491554, 2010.

[7] P. Memmolo, A. Finizio, M. Paturzo, L. Miccio, and P. Ferraro. Twin-beams digital holography for 3 d tracking and quantitative phasecontrast microscopy in microfluidics. Optics express, 19(25):25833-25842, 2011.

[8] E. Cuche, P. Marquet, and C. Depeursinge. Simultaneous amplitude-contrast and quantitative phase-contrast microscopy by numerical reconstruction of fresnel off-axis holograms. Applied optics, 38(34):6994-7001, 1999.
[9] Jeon Woong Kang, Niyom Lue, Chae-Ryon Kong, Ishan Barman, Narahara C Dingari, Stephen J Goldfless, Jacquin C Niles, Ramachandra R Dasari, and Michael S Feld. Combined confocal raman and quantitative phase microscopy system for biomedical diagnosis. Biomedical optics express, 2(9):2484-2492, 2011.

[10] Xiangyu Quan, Peng Xia, Osamu Matoba, Koichi Nitta, and Yasuhiro Awatsuji. Multimodal digital holographic microscopy for widefield fluorescence and $3 \mathrm{~d}$ phase imaging. In Quantitative Phase Imaging II, volume 9718, page 971821. International Society for Optics and Photonics, 2016.

[11] Benjamin Rappaz, Florian Charrière, Christian Depeursinge, Pierre J. Magistretti, and Pierre Marquet. Simultaneous cell morphometry and refractive index measurement with dualwavelength digital holographic microscopy and dye-enhanced dispersion of perfusion medium. Opt. Lett., 33(7):744-746, Apr 2008.

[12] B. Kemper and G. von Bally. Digital holographic microscopy for live cell applications and technical inspection. Applied optics, 47(4):A52-A61, 2008.

[13] Gabriel Popescu, YoungKeun Park, Niyom Lue, Catherine Best-Popescu, Lauren Deflores, Ramachandra R Dasari, Michael S Feld, and Kamran Badizadegan. Optical imaging of cell mass and growth dynamics. American Journal of Physiology-Cell Physiology, 295(2):C538-C544, 2008.

[14] M. Debailleul, V. Georges, B. Simon, R. Morin, and O. Haeberlé. High-resolution threedimensional tomographic diffractive microscopy of transparent inorganic and biological samples. Optics letters, 34(1):79-81, 2009.

[15] Y. Sung, W. Choi, C. Fang-Yen, K. Badizadegan, R.R. Dasari, and M.S. Feld. Optical diffraction tomography for high resolution live cell imaging. Optics express, 17(1):266-277, 2009. 
[16] D.L. Donoho. Compressed sensing. IEEE Transactions on information theory, 52(4):1289-1306, 2006.

[17] E.J. Candes, J.K. Romberg, and T. Tao. Stable signal recovery from incomplete and inaccurate measurements. Communications on Pure and Applied Mathematics: A Journal Issued by the Courant Institute of Mathematical Sciences, 59(8):1207-1223, 2006.

[18] D.J. Brady, K. Choi, D.L. Marks, R. Horisaki, and S. Lim. Compressive holography. Optics express, 17(15):13040-13049, 2009.

[19] L. Denis, D. Lorenz, E. Thiébaut, C. Fournier, and D. Trede. Inline hologram reconstruction with sparsity constraints. Optics letters, 34(22):3475-3477, 2009.

[20] Y. Rivenson, A. Stern, and B. Javidi. Compressive fresnel holography. Journal of Display Technology, 6(10):506-509, 2010.

[21] M.M. Marim, M. Atlan, E. Angelini, and J.C. Olivo-Marin. Compressed sensing with offaxis frequency-shifting holography. Optics letters, 35(6):871-873, 2010.

[22] K. Choi, R. Horisaki, J. Hahn, S. Lim, D.L. Marks, T.J. Schulz, and D.J. Brady. Compressive holography of diffuse objects. Applied optics, 49(34):H1-H10, 2010.

[23] J. Hahn, S. Lim, K. Choi, R. Horisaki, and D.J. Brady. Video-rate compressive holographic microscopic tomography. Optics express, 19(8):7289-7298, 2011.

[24] O. Cossairt, K. He, R. Shang, N. Matsuda, M. Sharma, X. Huang, A. Katsaggelos, L. Spinoulas, and S. Yoo. Compressive reconstruction for $3 \mathrm{~d}$ incoherent holographic microscopy. In 2016 IEEE International Conference on Image Processing (ICIP), pages 958962, Sep. 2016.

[25] Zihao Wang, Leonidas Spinoulas, Kuan He, Lei Tian, Oliver Cossairt, Aggelos K. Katsaggelos, and Huaijin Chen. Compressive holographic video. Opt. Express, 25(1):250-262, Jan 2017.

[26] D. Donnarumma, A. Brodoline, D. Alexandre, and M. Gross. 4d holographic microscopy of zebrafish larvae microcirculation. Optics express, 24(23):26887-26900, 2016.

[27] A. Brodoline, N. Rawat, D. Alexandre, N. Cubedo, and M. Gross. 4d compressive sensing holographic microscopy imaging of small moving objects. Optics Letters, 44(11):2827$2830,2019$.

[28] Stijn Bettens, Hao Yan, David Blinder, Heidi Ottevaere, Colas Schretter, and Peter Schelkens. Studies on the sparsifying operator in compressive digital holography. Opt. Express, 25(16):18656-18676, Aug 2017.

[29] Jing Liu, Guoxian Zhang, Kai Zhao, and Xiaoyu Jiang. Compressive holography algorithm for the objects composed of point sources. Appl. Opt., 56(3):530-542, Jan 2017.

[30] Monte Westerfield. The zebrafish book : a guide for the laboratory use of zebrafish (Danio rerio). University of Oregon press, 2007.

[31] Thorsten Schwerte and Bernd Pelster. Digital motion analysis as a tool for analysing the shape and performance of the circulatory system in transparent animals. Journal of Experimental Biology, 203(11):1659-1669, 2000.

[32] J. Gao, J.A. Lyon, D.P. Szeto, and J. Chen. In vivo imaging and quantitative analysis of zebrafish embryos by digital holographic microscopy. Biomedical optics express, 3(10):26232635, 2012.

[33] E. Cuche, P. Marquet, and C. Depeursinge. Spatial filtering for zero-order and twin-image elimination in digital off-axis holography. Applied optics, 39(23):4070-4075, 2000.

[34] T. Colomb, E. Cuche, F. Charrière, J. Kühn, N. Aspert, F. Montfort, P. Marquet, and C. Depeursinge. Automatic procedure for aberration 
compensation in digital holographic microscopy and applications to specimen shape compensation. Applied optics, 45(5):851-863, 2006.

[35] N. Verrier, D. Alexandre, G. Tessier, and M. Gross. Holographic microscopy reconstruction in both object and image half-spaces with an undistorted three-dimensional grid. Applied optics, 54(15):4672-4677, 2015.

[36] L. Yu and M.K. Kim. Wavelength-scanning digital interference holography for tomographic three-dimensional imaging by use of the angular spectrum method. Optics letters, 30(16):20922094, 2005.

[37] Y.C. Pati, R. Rezaiifar, and P.S. Krishnaprasad. Orthogonal matching pursuit: Recursive function approximation with applications to wavelet decomposition. In Signals, Systems and Computers., pages 40-44. IEEE, 1993.

[38] Sumio Isogai, Masaharu Horiguchi, and Brant M Weinstein. The vascular anatomy of the developing zebrafish: an atlas of embryonic and early larval development. Developmental biology, 230(2):278-301, 2001.

[39] D. Liu, J. Gu, Y. Hitomi, M. Gupta, T. Mitsunaga, and S. K. Nayar. Efficient spacetime sampling with pixel-wise coded exposure for high-speed imaging. IEEE Transactions on Pattern Analysis and Machine Intelligence, 36(2):248-260, Feb 2014.

[40] Zihao Wang, Qiqin Dai, Donghun Ryu, Kuan He, Roarke Horstmeyer, Aggelos K. Katsaggelos, and Oliver Cossairt. Dictionary-based phase retrieval for space-time super resolution using lens-free on-chip holographic video. In Imaging and Applied Optics 2017 (3D, AIO, COSI, IS, $M A T H, p c A O P)$, page CTu2B.3. Optical Society of America, 2017.

[41] Robert Tibshirani. Regression shrinkage and selection via the lasso. Journal of the Royal Statistical Society: Series B (Methodological), 58(1):267-288, 1996.
[42] Amir Beck and Marc Teboulle. A fast iterative shrinkage-thresholding algorithm for linear inverse problems. SIAM journal on imaging sciences, 2(1):183-202, 2009.

[43] S. Ji, Y. Xue, and L. Carin. Bayesian compressive sensing. IEEE Transactions on Signal Processing, 56(6):2346-2356, June 2008.

[44] A. Brodoline, N. Rawat, D. Donnarumma, D. Alexandre, N. Cubedo, and M. Gross. Compressive sensing holographic microscopy for imaging of sparse moving objects in 3d. In Digital Holography and Three-Dimensional Imaging 2019, page Th2B.7. Optical Society of America, 2019 . 